\title{
Editorial: Recent Advances in Continuous Cultivation
}

\author{
Manfred Zinn ${ }^{1 *}$, Thomas Egli ${ }^{2,3}$, Christoph Herwig ${ }^{4,5,6}$ and Atul Narang ${ }^{7}$ \\ ${ }^{1}$ Institute of Life Technology, School of Engineering, University of Applied Sciences Western Switzerland, Sion, Switzerland, \\ ${ }^{2}$ Department of Environmental Systems Science, Swiss Federal Institute of Technology, Zurich, Switzerland, \\ ${ }^{3}$ Microbes-in-Water GmbH, Feldmeilen, Switzerland, ${ }^{4}$ Christian Doppler Laboratory for Mechanistic and Physiological \\ Methods for Improved Bioprocesses, Vienna, Austria, ${ }^{5}$ Competence Center CHASE GmbH, Linz, Austria, ${ }^{6}$ Research Area \\ Biochemical Engineering, Institute of Chemical Engineering, Vienna, Austria, ${ }^{7}$ Department of Biochemical Engineering and \\ Biotechnology, Indian Institute of Technology Delhi, New Delhi, India
}

Keywords: chemostat, continuous culture, cell physiology, growth conditions, cultivation, growth medium, process analytical technology, growth kinetics

\section{Editorial on the Research Topic}

\section{Recent Advances in Continuous Cultivation}

The scientific work by Monod (1950) and Novick and Szilard (1950a,b) on continuous cultivation in the 1950s and their early analyses and applications by the "Porton" and the "Prague" groups (Herbert et al., 1956; Malek et al., 1958; Pirt and Callow, 1960; Herbert, 1961) evened the path toward a better understanding of the cell physiology of microorganisms. The chemostat, where all physicochemical growth conditions can be kept constant, soon became a very important research tool to study the nutritional needs as a function of the specific growth rate of microorganisms. The recent evolution of new disciplines in systems biology offering a plethora of novel, scientific methods (e.g., transcriptional analysis, proteomics, metabolomics, and detailed metabolic flux analysis) played an important role in gaining a better understanding of the influence of the cell physiology and product formation.

To date, industry is investing in the development of continuous production processes, because of their better volumetric productivity and hence lower equipment cost. Moreover, powerful methods have been developed for continuous medium preparation and product purification (e.g., isolation of recombinant proteins in simulated moving bed chromatography). Finally, a continuous process can be run for an extended period and thus results in a more desired, ideally time invariant, and reproducible product quality. In future, more continuous bioprocesses will be used to produce pharmaceuticals, since the American Food and Drug Administration recently approved drugs produced by continuous cultivation (Matsunami et al., 2018). This change has been made possible because of the good experiences with their initiative Quality by Design $(\mathrm{QbD})$ and the real-time control of cultivations using Process Analytical Technology (PAT).

The goal of this electronic article collection is to give an overview on recent scientific advances in continuous cultivation. In their review article, Nieto-Taype et al. summarize the potential of continuous cultivations of Pichia pastoris (Komagataella phaffii) with a particular focus on closed feed-back loop control using PAT. Typical examples are the turbidostat (cell density controlled), nutristat (nutrient concentration controlled), and among others, the $\mathrm{pH}$-auxostat, where the substrate consumption changes the $\mathrm{pH}$ and thus triggers a $\mathrm{pH}$ changing substrate feed. In general, the cells cultured under these conditions are growing at almost maximum specific growth rate $\left(\mu_{\max }\right)$. Other interesting applications of continuous cultivation are changestats, where the dilution rate is continuously increased (accelerostats or A-stats), decreased (deceleration-stats or De-stats), or kept constant with a continuous change of another parameter (e.g., medium composition). A large potential of producing secondary metabolites and products is the retentostat, where all biomass is retained in a bioreactor and where the specific growth rate is practically zero. 
Adamberg et al. applied the above described changestat approach to assess the adaptation of a human gut microbiota in A-stats and D-stats. The influence of the dilution rate (D) was tested either starting with steady-states at $D=0.05 \mathrm{~h}^{-1}$ or $D=$ $0.2 \mathrm{~h}^{-1}$ and increasing or decreasing the dilution rate to $D=$ $0.05 \mathrm{~h}^{-1}$ or $D=0.2 \mathrm{~h}^{-1}$, respectively. Interestingly, the detected population distribution was consistent and confirmed the fact that in continuous cultivation the Monod constant (Ks) plays a decisive role in the population dynamics. Finally, this finding is important to enable an industrial cultivation of multi-strain probiotics and fecal transplantation mixtures.

Barbera et al. describe the powerful approach of the chemostat cultivation to determine the necessary growth kinetics of the diazotrophic cyanobacterium Anabaena PCC7122t model growth. The traditional batch approach was not appropriate, since along with the increase of the cell density, the average illumination is reduced and thus the metabolism is significantly affected. However, using small photobioreactors operated in a continuous mode, Barbera et al. were able to determine $\mu_{\max }$ and the half saturation constant for nitrogen $\left(\mathrm{K}_{\mathrm{N}}\right)$. They also could show that the specific maintenance rate was influenced by the medium composition and the illumination intensity supplied to the culture.

Velu et al. investigated the potential of continuous outdoor cultures of the cyanobacterium Tolypothrix sp. by experimentally simulating the remediation of waste carbon dioxide (flue gas) and metal containing ash dam water from coal-fired power plants that are typically found in Australia. The entire process was economically feasible when the remediation was combined with the production of foodgrade phycocyanin alone or in combination with biomass production as biofertilizer using either vertical or raceway suspension cultures.

The potential of the acetogenic bacterium Clostridium autoethanogenum to generate biofuel and chemicals from $\mathrm{CO}_{2}$ and $\mathrm{H}_{2}$ was assessed by Heffernan et al. using chemostat cultivations. The authors combined their experimental approach with a metabolic flux model and were thus able to optimize the conversion efficiency by supplementing the culture with $\mathrm{CO}$. In future, this data set may be used to favor biosynthesis of a particular cell product.

Many physiological studies have shown that recombinant plasmids containing antibiotic resistance as selection marker may get lost without antibiotic selection, which renders this expression system failure prone and more expensive because of the need for continuous addition of antibiotics. However, new techniques, like CRISPR/CAS9 and related methods guarantee a better genetic stability. Nevertheless, the natural selection of better adapted cells (natural mutants; Novick and Szilard, 1950b) is leading to change in population distribution favoring the cells with a lower genetic burden. Because of such a population shift, the productivity may significantly be reduced or even lost completely. Kopp, Slouka et al. propose to evaluate the dynamic feeding and selection that takes place in the recently developed segregostat and also give an overview on existing online monitoring tools for Escherichia coli.

In industry, the product synthesis is frequently done in fed-batch cultivations in a phase separated manner, meaning the biomass formation is followed by the induction of genes of interest during the production phase. This set-up can also be established in a continuous manner by using twostage chemostats. Kopp, Kolkmann et al. exemplified such recombinant protein production (RPP) in Escherichia coli in a two-stage chemostat using glycerol as a carbon source in the first stage and glycerol and lactose as inducers replacing IPTG in the second stage. The space-time-yield could be increased by a factor of 100 in comparison to a single-stage chemostat cultivation.

This continuous two-stage cultivation technique has also been implemented in research studies. Hanik et al. reported the production of tailor-made medium chain length poly(3hydroxyalkanoate) (mcl-PHA) in two-stage, parallel chemostat cultivations. In the first stage (one bioreactor) Pseudomonas putida was grown and in the second chemostat stage consisting of 4 parallel chemostats the substrates for mcl-PHA production (phenylvaleric and decanoic acids) were added. This approach allowed a faster identification of the design window for tailormade PHA production.

Tapia et al. developed a two-stage chemostat cultivation technique in order to assess the viral dynamics and the interaction of defective interfering particles (DIPs) of influenza A virus with the canine cell line MDCK.SU2. The design of a mathematical model enabled the interpretation of the observed cell and viral oscillations in the second chemostat stage. It was concluded that continuous two-stage cultivations could be efficiently used to produce DIPs.

\section{AUTHOR CONTRIBUTIONS}

MZ has drafted the manuscript and submitted in final form. $\mathrm{TE}, \mathrm{CH}$, and AN have revised the manuscript draft. All authors contributed to the article and approved the submitted version.

\section{FUNDING}

This work was financed by the University of Applied Sciences and Arts Western Switzerland, the Swiss Universities, the Christian Doppler Society, Austria, Microbes-in-water, Switzerland, and by the Department of Science and Technology, India.

\section{ACKNOWLEDGMENTS}

The Editors thank all authors who have contributed to this special issue. We are happy for being able to document with this collection of articles the latest promising scientific advancement to leverage the large potential of continuous cultivation and to finally assess, control, and optimize product formation in bioprocesses. 


\section{REFERENCES}

Herbert, D. (1961). A theoretical analysis of continuous culture systems. Soc. Chem. Industry Monogr. 12, 21-53.

Herbert, D., Elsworth, R., and Telling, R. C. (1956). The continuous culture of bacteria; a theoretical and experimental study. Microbiology 14, 601-622. doi: 10.1099/00221287-14-3-601

Malek, I. (1958) “The physiological state of microorganisms during continuous culture" in Continuous Cultivation of Microorganisms: A Symposium (Prague: Publishing House of the Czechoslovak Academy of Sciences).

Matsunami, K., Miyano, T., Arai, H., Nakagawa, H., Hirao, M., and Sugiyama, H. (2018). Decision support method for the choice between batch and continuous technologies in solid drug product manufacturing. Ind. Eng. Chem. Res. 57, 9798-9809. doi: 10.1021/acs.iecr.7b05230

Monod, J. (1950). La technique de culture continue; théorie et applications. Ann. Inst. Pasteur 79, 390-410.

Novick, A., and Szilard, L. (1950a). Description of the chemostat. Science 112, 715-716. doi: 10.1126/science.112.2920.715
Novick, A., and Szilard, L. (1950b). Experiments with the chemostat on spontaneous mutations of bacteria. Proc. Natl. Acad. Sci. 36, 708-719. doi: 10.1073/pnas.36.12.708

Pirt, S. J., and Callow, D. S. (1960). Studies of the growth of Penicillium chrysogenum in continuous flow culture with reference to penicillin production. J. Appl. Bacteriol. 23, 87-98. doi: 10.1111/j.1365-2672.1960.tb00181.x

Conflict of Interest: The authors declare that the research was conducted in the absence of any commercial or financial relationships that could be construed as a potential conflict of interest.

Copyright (C) 2021 Zinn, Egli, Herwig and Narang. This is an open-access article distributed under the terms of the Creative Commons Attribution License (CC BY). The use, distribution or reproduction in other forums is permitted, provided the original author(s) and the copyright owner(s) are credited and that the original publication in this journal is cited, in accordance with accepted academic practice. No use, distribution or reproduction is permitted which does not comply with these terms. 